\title{
Catch me if you can - Das endobronchiale Lipom
}

\author{
Catch Me If You Can - A Case of Endobronchial Lipoma
}

Autoren

Institute
H. Knoop ${ }^{1}$, U. Knoop ${ }^{2}$, L. Heining ${ }^{1}$, T. Magin ${ }^{3}$, G. Richardsen ${ }^{4}$, R. Wolf' ${ }^{1}$ S. Ewig'

Die Institutsangaben sind am Ende des Beitrags gelistet. eingereicht 21.3. 2012 akzeptiert nach Revision 22. 3.2012

\section{Bibliografie}

Dol http://dx.doi.org/

10.1055/s-0032-1309397

Online-Publikation: 24.4.2012

Pneumologie 2012; 66: 558-559

(c) Georg Thieme Verlag KG

Stuttgart · New York

ISSN 0934-8387

\section{Korrespondenzadresse}

\section{Dr. med. Heiko Knoop}

Thoraxzentrum Ruhrgebiet Klinik für Pneumologie und Infektiologie

Evangelisches Krankenhaus Herne - Betriebsstelle Eickel Akademisches Lehrkrankenhaus der Universität Duisburg-Essen

Hordeler Str. 7 - 9

44651 Herne

Heiko.Knoop@web.de

\section{Zusammenfassung \\ $\nabla$}

Bei einem 74-jährigen Patienten fiel akzidentell eine Raumforderung im rechten Oberlappen auf. Im Rahmen der Abklärung zeigte sich als Zufallsbefund bronchoskopisch ein endobronchiales Lipom im rechten Unterlappen, das zunächst belassen wurde. Angesichts der vorhandenen Komorbiditäten erfolgte zunächst eine Verlaufsbeobachtung. Aufgrund eines Größenwachstums mit drohender Atemwegsverlegung wurde das endobronchiale Lipom schließlich mittels Kryoextraktion entfernt. Dieser Fallbericht über ein endobronchiales Lipom gibt eine kurze Übersicht über diesen seltenen gutartigen Lungentumor.

Im Juni 2011 wurde bei einem 74-jährigen Patienten im Rahmen einer gefäßchirurgischen Behandlung in einem auswärtigen Krankenhaus in der Röntgen-Thoraxaufnahme eine Raumforderung im rechten Oberlappen gesehen. Die weitere Abklärung dieser Veränderung erfolgte zunächst ebenfalls auswärtig: Die angeschlossene CT-Thorax zeigte einen $9 \mathrm{~mm}$ durchmessenden, unscharf begrenzten Rundherd peripher im Segment 1 mit feinen Ausziehungen. Eine Histologie konnte endoskopisch nicht gewonnen werden. Allerdings fiel als Zufallsbefund im rechten Unterlappen (RB9) eine polypöse Struktur auf. Die Histologie dieser Struktur zeigte ein endobronchiales Lipom. Aufgrund der Dignität des Befundes und fehlender Hinweise auf eine Verlegung des Bronchus durch das Lipom wurden diesbezüglich keine weiteren Maßnahmen ergriffen. Zur Mitbeurteilung der Raumforderung im rechten Oberlappen wurde der Patient im August 2011 erstmalig in unserer Thoraxchirurgie vorgestellt. Die Kollegen diskutierten angesichts eines Risikoprofils von 40 pack-years eine definitive chirurgische Klärung der Raumforderung im rechten Oberlappen. Letztlich fiel die Entscheidung leitlinienkonform [1] und vor dem Hintergrund zahlreicher Komor-

\section{Abstract \\ $\nabla$}

Incidentally, a lesion in the right upper pulmonary lobe was found in a 74-year-old male patient. The flexible bronchoscopy revealed an endobrochial lipoma in the right lower lobe as the only pathological finding. Due to multiple comorbidities, no intervention was performed initially. Since the endobronchial lipoma increased in size during follow-up, it was removed by cryoextraction. This case report on an endobronchial lipoma includes a short summary of this rare benign lung tumor.

biditäten (koronare 3-Gefäßerkrankung, permanentes Vorhofflimmern, COPD, chronische Niereninsuffizienz im Stadium 2, pAVK Stadium IV, arterielle Hypertonie) zugunsten regelmäßiger Verlaufskontrollen. Computertomografisch zeigte der Herd im rechten Oberlappen bis zuletzt keine Dynamik (s. $\odot$ Abb. 1) In der flexiblen Bronchoskopie fiel allerdings eine Größenzunahme der als Lipom vorbeschriebenen Struktur mit drohender Verlegung des Ostiums von RB9 auf (s. @ Abb.2), sodass wir den Patienten stationär zur starren Bronchoskopie mit Lipomabtragung aufnahmen. Diese erfolgte komplikationslos mittels Kryoextraktion mit nur mäßiger Nachblutung. Das polyzyklisch konfigurierte, glatt begrenzte Lipom konnte nahezu vollständig abgetragen werden. Eine bronchoskopische und CT-Thorax Kontrolle ist in 3 Monaten erneut vorgesehen.

Pulmonale Lipome sind seltene gutartige Tumoren, die $0,01 \%$ aller Lungentumoren und weniger als $5 \%$ der benignen Lungentumoren darstellen [2]. Sie sind wenig vaskularisiert [3], wachsen langsam und haben ein niedriges Entartungsrisiko [4]. Die häufigste Komplikation durch endobronchiale Lipome ist die bronchiale Verlegung mit konsekutiver poststenotischer Pneumo- 


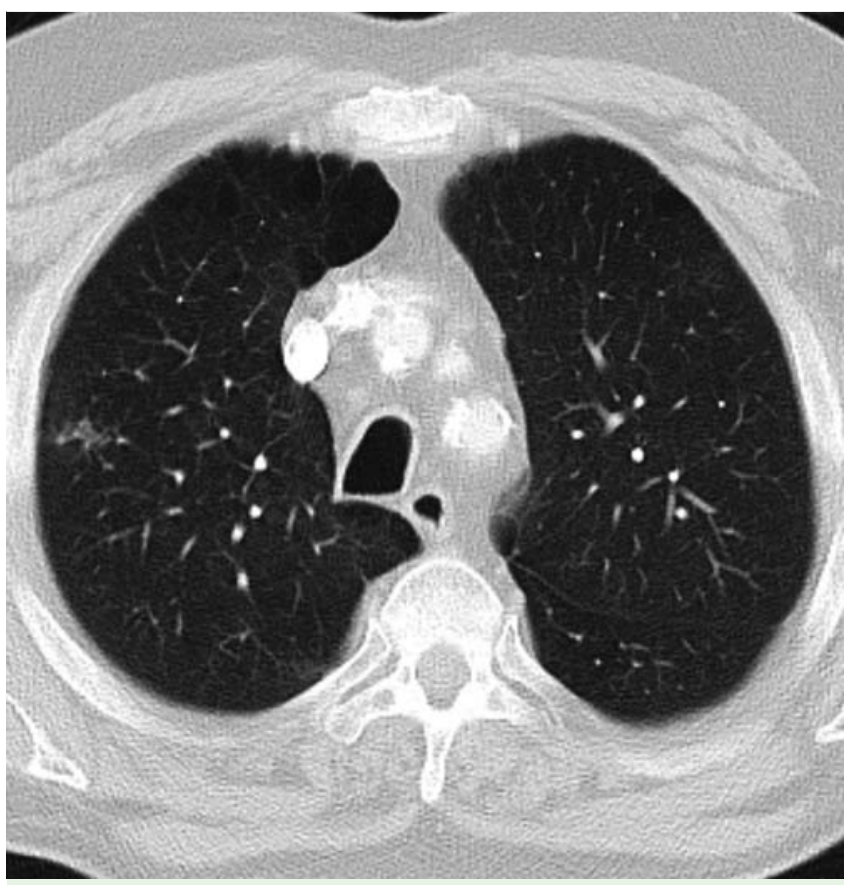

Abb. 1 Computertomografie des Thorax mit Kontrastmittelgabe mit Nachweis einer $9 \mathrm{~mm}$ durchmessenden, unscharf begrenzten Struktur peripher im Segment 1 mit feinen Ausziehungen.

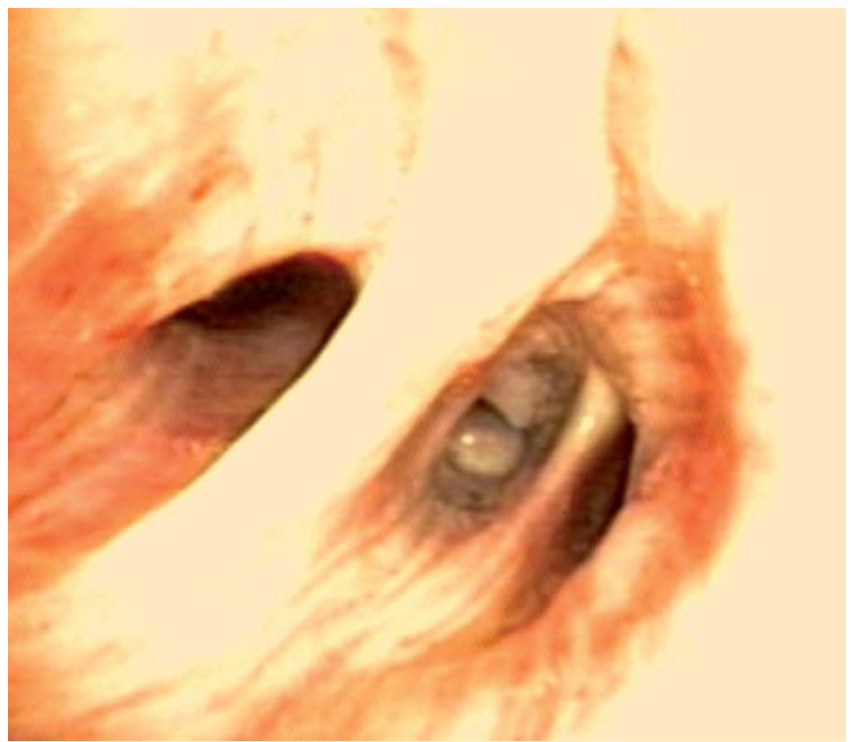

Abb. 2 Glatt begrenzte, polyzyklisch konfigurierte polypöse Struktur im rechten Unterlappen (Segment 9), das Ostium nahezu komplett verlegend.

nie und gelegentlich auch Bronchiektasenbildung [5]. Die poststenotische Pneumonie bestimmt das klinische Erscheinungsbild aus trockenem oder produktivem Husten, Thoraxschmerzen, Luftnot und Fieber. Ferner wurden im Kontext poststenotischer Pneumonien durch endobronchiale Lipome Hämoptysen beschrieben [6]. Risikofaktoren, die zur Entstehung dieser Tumorart führen, sind nicht eindeutig identifiziert. Inhalatives Zigarettenrauchen und Adipositas werden am häufigsten in der Literatur genannt [7]. Therapeutisch kommt neben der operativen Resektion die endoskopische Abtragung mittels Laser, Kryotherapie und Elektrokauter infrage [7-10]. Die Entscheidung, welche endoskopische Technik zum Einsatz kommt, ist individuell und auch von lokalen Gegebenheiten abhängig. Die Methoden sind als etwa gleichwertig anzusehen, eine Standardtherapie ist nicht definiert [7]. Das Risiko eines Rezidivs ist als gering einzuschätzen [2]. Eine Therapie ist aufgrund der drohenden Atemwegsobstruktion - wie in unserem Fall - bereits bei asymptomatischen Patienten indiziert [11]. Bei permanentem Vorhofflimmern und einem $\mathrm{CHA}_{2} \mathrm{DS}_{2}$-VASc-Score von 3 sollte leitliniengerecht [12] auch vor dem Hintergrund des geringen Risikos von Hämoptysen/-toe bei Patienten mit endobronchialem Lipom nicht auf eine Antikoagulation verzichtet werden.

\section{Interessenkonflikt}

\section{$\nabla$}

Die Autoren geben an, dass kein Interessenkonflikt besteht.

\section{Institute}

${ }^{1}$ Thoraxzentrum Ruhrgebiet, Evangelisches Krankenhaus Herne Betriebsstelle Eickel, Klinik für Pneumologie und Infektiologie (Chefarzt: Prof. Dr. med. S. Ewig)

2 Medizinische Klinik III - Pneumologie, Allergologie, Schlaf- und Beatmungsmedizin, Berufsgenossenschaftliches Universitätsklinikum Bergmannsheil GmbH (Direktor: Prof. Dr. med. J. Behr)

${ }^{3}$ Radiologie Herne (Leitender Arzt: Dr. med. T. Magin)

4 Thoraxzentrum Ruhrgebiet, Evangelisches Krankenhaus Herne -

Betriebsstelle Eickel, Klinik für Thoraxchirurgie (Chefarzt: Dr. med. Dipl.-Oec. E. Hecker)

\section{Literatur}

1 Goeckenjan G, Sitter H, Thomas M et al. Prävention, Diagnostik, Therapie und Nachsorge des Lungenkarzinoms. Pneumologie 2010; 64: 23 155

2 Shah H, Garbe L, Nussbaum E et al. Benign tumors of the tracheobronchial tree. Endoscopic characteristics and role of laser resection. Chest 1995; 107: 1744-1751

3 Yokozaki M, Kodama T, Yokose T et al. Endobronchial lipoma associated with lobar bronchiectasis. Respir Med 1991; 85: 71 - 72

4 Muraoka M, Oka T, Akamine S et al. Endobronchial lipoma: review of 64 cases reported in Japan. Chest 2003; 123: 293-296

5 Filosso PL, Giobbe R, Mossetti C et al. Hemoptysis caused by an endobronchial lipoma. J Thorac Vasc Surg 2008; 135: 954-955

6 Cao D, Sun Y, Yang S. Endobronchial lipoma: an unusual cause of bronchial obstruction. Case Report Med 2011: DOI 10.1155/2011/939808

7 Nassiri AH, Dutau H, Breen D et al. A multicenter retrospective study investigating the role of interventional bronchoscopic techniques in the management of endobronchial lipomas. Respiration 2008; 75: $79-84$

8 Lamprecht B, Hutarew G, Porsch P et al. Successful bronchoscopic cryorecanalization in a case of endobronchial lipoma. Diagn Ther Endosc 2011: DOI $10.1155 / 2011 / 845686$

9 Huisman C, van Kralingen KW, Postmus PE et al. Endobronchial lipoma: a series of three cases and the role of electrocautery. Respiration 2000; 67: 689-692

10 Franke KJ, Nilius G, Rühle $\mathrm{KH}$. Kryotherapie eines endobronchialen Lipoms. Pneumologie 2005; 59: 685-688

11 Krüger S, Stanzel F, Morresi-Hauf A et al. Endobronchiales Lipom: erfolgreiche Therapie mittels bronchoskopischer Laserresektion versus Chirurgie. Pneumologie 2004; 58: 769-772

12 Camm AJ, Kirchhof P, Lip GY et al. Guidelines for the management of atrial fibrillation: the Task Force for the Management of Atrial Fibrillation of the European Society of Cardiology (ESC). Europace 2010; 12: $1360-1420$ 